\title{
Über den Aktionsstrom der neurogenen und myogenen Kontraktion des Limulusherzens.
}

\author{
Von \\ Daizo Ogata und Kazuo Șuenaga. \\ (緒方大象) (末永一男)
}

(Aus dem I. Physiologischen Institut, Medizinische Fakultät, Kyusyu Kaiserliche Universität zu Hukuoka.

Vorstand: Prof. D.Ogata.)

\section{Einleitung.}

Im hiesigen physiologischen Institut wurde seit Jahren die Automatie des Limulusherzens untersucht, und es wurde klargestellt, dass das Limulusherz normalerweise neurogen schlägt. Andererseits stellten $\mathrm{Hosh} \mathrm{in}^{5)}$ u.a. fest, dass das Herz nach Exstirpation des medianen Nervenstrangs noch weiter fortzuschlagen befähigt ist, d.h. die myogene Kontraktion zeigt.

Das Limulus-Ekg, richtiger Eg, wurde zuerst von Hoff mann ${ }^{4)}$ (1911), dann von Nukada ${ }^{7)}$ (1917), Dubuisson ${ }^{1)}$ (1930), Rijlant ${ }^{8)}$ (1931), Garry ${ }^{2)}(1932)$ und Hein becker) (1933) photographisch registriert. Es beschränkt sich jedoch ausschliesslich auf die neurogene Kontraktion und bezog sich niemals auf die sogenannte myogene Kontraktion.

\section{Materialien und Methodik.}

Die Materialien, die für Verff. zur Untersuchung gestellt wurden, wurden in der See nahe Hukuoka, insbesondere in der Umgebung der Noko Insel gesammelt. Im ganzen wurden 20 Tiere, sowohl männliche wie auch weibliche benutzt, die von 500-2300 g wogen. Die Elektroden waren gewöhliche Platindrähte. Der Aktionsstrom wurde mittels elektromagnetischer Oscillographen (Yokogawa) nach Verstärkung registriert. Die maximale Empfindlichkeit war $2 \mathrm{~cm}$ Anschlag für $0,1 \mathrm{mV}$.

Die Elektroden wurden meistenteils auf das 2. Segment des Herzens, manchmal in der Längs-, manchmal in der Querrichtung gelegt. 
In einigen Fällen wurde die monopolare Ableitung ausgeführt, d.h. die eine der beiden Elektroden wurde direkt auf das Herz gelegt, während die andere auf die umgebenden Gewebe ausser dem Herzen gelegt wurde. Bei der monopolaren Ableitung war die potentiale Veränderung im allgemeinen höher als bei der bipolaren Ableitung.

3. Die Versuchsergebnisse.

i

Die Kurve vom Herzen in situ weist ohne weiteres auf eine tetanische Natur hin. Für einzelne Kontraktion sieht man jedesmal eine grosse Welle, worauf eine Serie von kleinen Wellen sich aufsetzt. Die grösseren Wellen treten immer in derselben Frequenz wie die des Herzschlags selbst auf. Sie beträgt 23-26 für eine Minute, durchschnittlich 24. Die Potentiale sind 0,2-0,35 Volt, durchschnittlich 0,27 Volt.

Die kleinen Wellen kommen 14-20 mal während einer Sekunde vor, durchschnittlich 16 mal, und die Potentiale schwanken von $0,01-$ 0,075 Volt, durchschnittlich 0,04 Volt. (Fig. 1.)

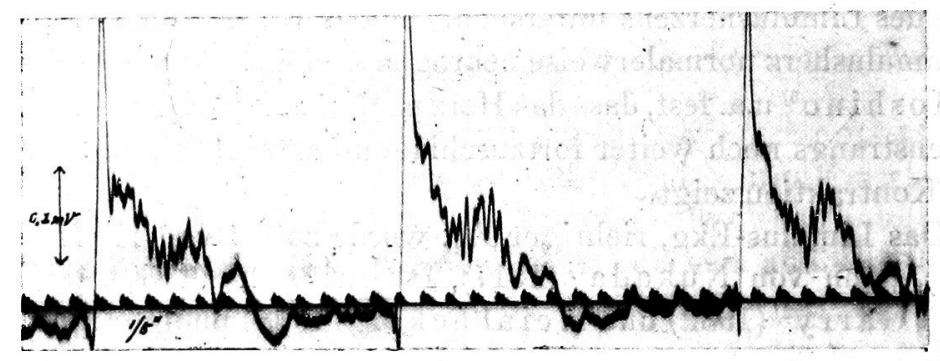

Fig. 1. Aktionsströme des Limulusherzens in situ. 3 grosse Wellen und darauf sitzende mehrere kleine Wellen. Zeit in $1 / 5$ Sekunde.

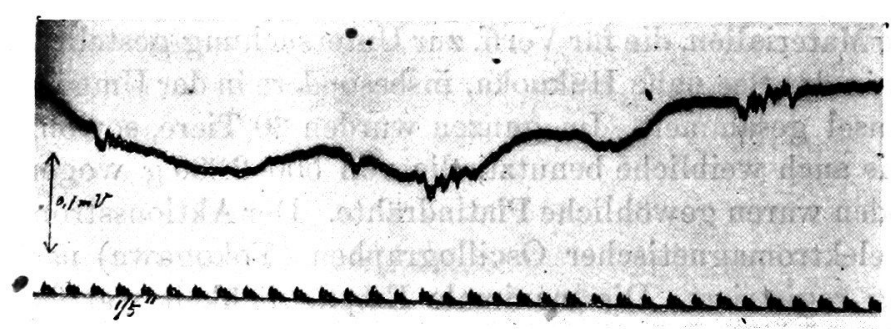

Fig. 2. Aktionsströme vom herausgeschnittenen medianen Nervenstrang des Limulusherzens. 3 Aktionsströme ersichtlich.

Zeit in $1 / 5$ Sekunde. 
Dann wurde der Aktionsstrom vom herausgeschnittenen medianen Nervenstrang allein untersucht. Infolge der kleinen Zahl der Versuche kann man leider von der Grösse der Potentiale nur wenig mit Bestimmtheit aussagen. Soweit ist es aber sicher, dass die grosse Welle fehlt, und die einzig vorhandenen kleinen Wellen kommen in der Frequenz von 17,5-27,5 für eine Sekunde, durchschnittlich 22,74 vor, und ihre Potentiale betragen ungefähr 0,01-0,025 Volt, durchschnittlich 0,017 Volt. (Fig. 2.)

Diese Ziffern übertreffen die des Herzens in situ in der Wellenzahl, aber stehen in den Potentialen etwas nach. Wenn man den grossen Unterschied der äusseren Bedingungen der beiden Fälle bemerkt, so sind diese Differenzen eher gering, und gerade diese mehr oder weniger deutliche Übereinstimmung gibt einen Anhaltspunkt für die neurogene Kontraktion des normalen Herzens.

Endlich wurde das Herz, von dem der mediane Nervenstrang entfernt wurde, zur Untersuchung benutzt. An solchem Herzen kann man manchmal einen spontanen Herzschlag beginnen sehen. Wenn es nicht der Fall ist, sind einige elektrische Anstösse schon genug, um den Schlag wieder zu verursachen:

Die Kontraktion ist in diesem Falle immer eine rhythmische, und die typische Tetanuskurve ist kaum zu finden. Meistenteils ist eine Kontraktion von einer grossen Welle begleitet. Die in Fig. 3 aufgezeichnete Kurve zeigt etwa 24 Wellen für eine Minute, und diese Frequenz stimmt mit der von einem normalen Herzen beinahe überein.

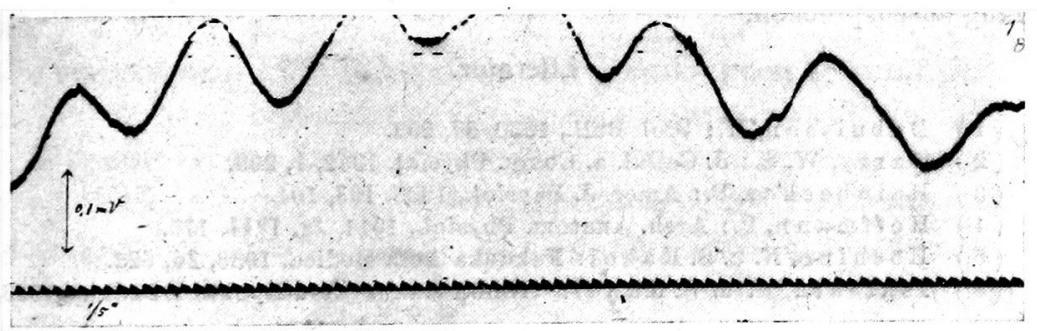

Fig. 3. Aktionsströme der myogenen Kontraktion des Limulusherzens. 6 diphasische Aktionsströme sichtbar, von denen einige die Grenze der Schreibfläche überschritten haben und durch die punktierte Linie teilweise ersetzt werden.

Zeit in $1 / 5$ Sekunde.

Jede Welle, genauer betrachtet, ist sicherlich eine Zuckung und die Potentiale liegen zwischen 0,025-0,075 Volt, durchschnittlich 0,05 Volt. 
Durch Exstirpation des medianen Nervenstrangs werden nach Hoshino und $\mathrm{Kakei}^{\mathrm{5})}$ die Ganglienzellen beinahe vollständig mit entfernt, so dass die darauf auftretende Kontraktion nicht anders als eine myogene sein kann. Die ähnliche Veränderung, d.h. der U̇bergang von einer neurogenen zu einer myogenen, ist von Verff. an dem Lymhherzen vor kurzem sicher nachgewiesen, wobei die Kontraktion sich von einem Tetanus zu einer Zuckung verändert. Daraus geht hervor, dass beim Limulusherzen die neurogene Kontraktion zur myogenen wechseln kann.

\section{Schluss.}

1. Das Limulusherz kann auch nach Exstirpation des medianen Nervenstrangs weiter zu schlagen fortsetzen.

2. Das Limulusherz in situ zeigt einen Aktionsstrom, der dem Tetanus entspricht, während das Herz, von dem der mediane Nervenstrang vollständig entfernt wird, einen Aktionsstrom zeigt, der der Zuckung entspricht.

3. Der herausgeschnittene mediane Nervenstrang selbst zeigt einen Aktionsstrom, der ebenfalls dem Tetanus gleichkommt.

4. Man kann daraus schliessen, dass der normale Schlag neurogen und von tetanischer Natur, und die myogene Kontraktion von zuckender Natur ist.

Wir gestatten uns, dem Unterrichtsministerium unseren besten Dank für die Gewährung einer Unterstützung zur Anregung wissenschaftlicher Forschung auszusprechen.

\section{Literatur.}

(1) D u bu is s o n, M.: Biol. Bull., 1930, 59, 293.

(2) Garry, W. S.: J. Cellul. a. Comp. Physiol. 1932, 1, 209.

(3) Hein becker, P.: Amer. J. Physiol., 1933, 103, 104.

(4) Hoffman n, P.: Arch. Anatom. Physiol., 1911, Jg. 1911, 175. -

(5) Hoshino, N. u. S. Kakei: Fukuoka Acta Medica, 1933, 26, 622. 498.

(6) Ishihara, M. u. S. Kakei: "Nihon Gakuzyutsu-Kyokai Hōkoku",1933, 8,

( 7) Nukada, S.: Mitteilungen aus der Med. Fakultät der Kais. Univ. Tokyo. $1918,19,1$.

(8) Rijlant, P.: C. R. Soc. Biol. 1931, 108, 825.

(9) 0 gata, D. u. K. Suenaga: Tohoku J. Ep. Med., 1941, 41, 92. 